\title{
Los desplazamientos forzados como riesgos sociales asociados a las condiciones de la violencia política en Colombia
}

\section{Resumen}

En territorio colombiano los desplazados delimitan una categoría migratoria peculiar, pues se sitúan en lo provisorio y están enredados por múltiples incertezas. Este artículo presenta una caracterización de la ambigüedad del sistema de protección social y la política social de mitigación de la pobreza entre la población en situación de desplazamiento. Las causas del fenómeno tienen que ver con las desigualdades persistentes y los grupos al margen de la ley para lograr el control de tierras en zonas estratégicas para el narcotráfico, poder político, tráfico de armas y contrabando. Los reiterados vacíos y fracasos en la atención a la población desplazada evidencian la incapacidad del Estado Social de Derecho en el proceso de consolidación de la política social como estrategia institucional.

Palabras-clave: Desplazamiento. Riesgos sociales. Colombia. Violencia. Política social.

\footnotetext{
Universidade do Vale do Rio dos Sinos (Unisinos), São Leopoldo, Rio Grande do Sul, Brasil.

** Pontifícia Universidade Javeriana de Bogotá, Bogotá, Colômbia.
} 


\section{Forced displacements as social risks related to the context of political violence in Colombia}

\section{Abstract}

In Colombian territory, displaced people comprise a peculiar category of migrants, since they live under a provisory condition and are entangled in multiple uncertainties. This article presents a characterization of the ambiguity involving the social protection system and the social policy aimed at mitigating poverty among the displaced population. The causes of the phenomenon are related to persistent inequalities and to the action of illegal groups for taking control of territories in strategic areas for drug trafficking, political power, and arms trafficking and smuggling. The repeated failures and omissions in relation to the displaced population demonstrate the inability of the Social State of Law (Estado Social de Derecho) for consolidating the social policy as an institutional strategy.

Keywords: Displacement. Social risks. Colombia. Violence. Social policy.

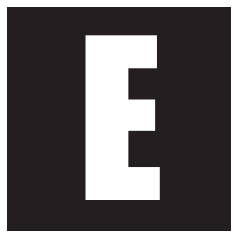

ste artículo se concentra particularmente en indicadores que reflejan los efectos sociales y las consecuencias de más de una década de incertezas ante el Estado Social de Derecho y la identificación de las nuevas vulnerabilidades en una estratificación social muy desigual que enfrenta la sociedad colombiana, a saber: el aumento del desempleo e informalidad laboral, persistencia de la pobreza e indigencia sin nuevas luces sobre cambios del capital cultural y el crecimiento de los grupos vulnerables como los desplazados por el conflicto armado interno.

Las familias desplazadas por la violencia viven cotidianamente los horrores de la fuga desesperada para escapar y esquivarse de la muerte y de las amenazas constantes en los territorios ocupados por los paramilitares o por el narcotráfico. En territorio colombiano los 'desplazados' delimitan 
una categoría migratoria peculiar, pues se sitúan en la condición de lo provisorio y están enredados por incertezas múltiples. La igualdad ante la ley es un principio de la Constitución Política de Colombia, de 1991, y de los Derechos Humanos, pero no se puede desconocer la desigualdad y la desventaja social real de los grupos excluidos de las esferas del poder económico y político. Por cierto, hay evidencias que muestran la sociedad como cerrada y selectiva, sin aversión a la iniquidad y desigualdad, lo que suscita discriminación de los pobres extremos, indigentes y población desplazada.

En el caso colombiano, los índices de violencia del conflicto armado, paramilitares y el narcotráfico, como una violencia pluriforme se han instalado en el centro de la agenda pública. El significativo incremento en los índices de violencia por el desplazamiento forzado, de acuerdo con Velásquez y Pinzón (2008), llevó a que la seguridad dejara el común tratamiento marginal y fragmentado para convertirse en una problemática prioritaria y generadora de articulaciones para la implementación de políticas públicas específicas y estrategias en la perspectiva de la seguridad ciudadana y convivencia.

Los procesos de atención y protección social son concebidos como una herramienta que, de un lado, es respuesta a problemáticas socialmente relevantes, de otro, radica en la legitimación y reproducción del orden político o de la gestión del Estado como el espacio de contacto entre las autoridades y los excluidos por medio de las políticas públicas. Desde este punto de vista, hay siempre un interrogante o sospecha de que el Estado nacional, por medio de sus desdoblamientos con alianzas políticas e intereses económicos difusos, posee compromisos tanto con la protección social de los ciudadanos, cuanto con las formas de ocasionar la desprotección social.

Si bien desde 1997 se ha venido desarrollando e implementando un amplio marco normativo, legislativo y judicial en torno al tema del des- 
plazamiento forzado, que configura los lineamientos de gestión pública en busca de la prevención, la atención integral y la estabilización socioeconómica de la población desplazada, el Estado colombiano incurre en contradicciones, que se evidencian en los compromisos políticos y en la fragilidad que caracteriza la atención del gobierno nacional.

\section{La consolidación del desplazamiento por la violencia del conflicto armado}

El desplazamiento forzado en Colombia no es un fenómeno nuevo, y de hecho, es complejo y de carácter casi permanente debido a las múltiples dinámicas sociales, económicas y políticas que lo vienen provocando. Desde el inicio del siglo XX, en el país, se han desarrollado conflictos y procesos sociales que han conducido a la expulsión y migración para la urbanización; según Escobar (2004), desde conflictos por el territorio y sus materiales naturales, de engendramiento de la invisibilidad de sectores empobrecidos, de apartados y formación de grupos ideológicos, hasta la violencia en la actualidad que enfrenta Colombia; enmarcada en la producción y comercialización ilegal de sustancias psicoactivas (Molano, 2007). El artículo 1o de la ley 387 de 1997, establece como desplazado:

Toda persona que se ha visto forzada a migrar dentro del territorio nacional abandonando su localidad de residencia o actividades económicas habituales, porque su vida, su integridad física, su seguridad o libertad personales han sido vulneradas o se encuentran directamente amenazadas, con ocasión de cualquiera de las siguientes situaciones: conflicto armado interno, disturbios y tensiones internas, violencia generalizada, violaciones masivas de los derechos humanos, infracciones al derecho internacional humanitario u otras circunstancias emanadas de las situaciones anteriores que puedan alterar o alteren drásticamente el orden público. 
Existen pocas señales de la reducción de los desplazamientos a lo largo de la década, pues los actores armados de acciones irregulares continúan generando nuevos refugiados. La situación colombiana al final de la década del 90 no es propiamente una novedad desconocida de la gestión pública. Todavía, existen evidencias del fenómeno de desplazamiento forzado, manifestado en el surgimiento de nuevas fuerzas sociales, con otros desafíos en las perspectivas de afirmaciones de interés público y su confrontación con intereses privados. Se comprenden los desplazamientos como nuevas dinámicas de la relación entre lo público y lo privado, como objeto de disputa por derechos y políticas públicas sectoriales. Para el mismo Barrios (2005), la emergencia de políticas de asistencia a los desplazados caracteriza algunos rasgos en la implementación de políticas para un determinado público vulnerable, a partir de las perversas acciones de grupos de interés particulares, acentuando las dificultades inherentes a la acción por la ciudadanía en el espacio gubernamental.

La magnitud estimada del desplazamiento forzado interno en Colombia en un período reciente, de acuerdo con las cifras de la entidad pública de Acción Social, asciende a un total de 3.470.387 personas y 794.523 hogares desplazados entre 1997 y 2010, de acuerdo con el Registro Único de Población Desplazadas (RUPD) ${ }^{1}$.

Según las cifras oficiales, manejadas por la Agencia Presidencial para la Acción Social y la Cooperación Social, con corte a 10 de abril de 2009, se informa que 2.938.349 personas (662.892 hogares) han sido registradas como víctimas del desplazamiento forzado. El mayor pico en la historia reciente del país, se registró durante el 2002, cuando se dio la mayor

\footnotetext{
${ }^{1}$ El RUPD, constituye una herramienta indispensable en el contexto de la atención integral porque identifica y reconoce a la población víctima del desplazamiento y hace un seguimiento a la evolución en sus diferentes componentes de atención. Así mismo, permite identificar las características propias de la población víctima del desplazamiento al tener en cuenta las condiciones sociodemográficas, culturales, étnicas y geográficas ... (Velásquez, 2011, p. 32).
} 
actividad armada de los grupos ilegales, desde el siguiente año a la actualidad se ha mantenido relativamente constante, con un promedio anual de 250.000 personas expulsadas de sus territorios. Aún así, actualmente no se encuentran disponibles las cifras oficiales sobre las características sociodemográficas de la población desplazada en Colombia incluidas en el RUPD.

Estas cifras presentan en su totalidad una abismal diferencia frente a las estimaciones del CODHES (Consultoría para los Derechos Humanos y el Desplazamiento) ${ }^{2}$. Además de las cifras globales y por período de las personas y los hogares desplazados en el país, las estadísticas oficiales ofrecen datos que determinan algunos rasgos característicos del como los siguientes: las tendencias de crecimiento, las zonas de expulsión, las zonas de recepción —-municipios en los cuales se han presentado declaraciones de las victimas - y las variaciones comparativas de expulsión, llegada, declaración y valoración.

Teniendo en cuenta lo anterior, y con miras a presentar un panorama mucho más amplio del fenómeno del desplazamiento forzado interno en Colombia, se expondrán algunas cifras publicadas que constituye un registro sólido de gran aceptación en el país, gracias a su metodología de fuentes contrastadas. Según este informe del año 2009 existe sobre todo una continuidad del proceso histórico:

Durante 2008 ocurrieron 82 eventos de desplazamientos masivos en 19 departamentos del territorio nacional [...] Alrededor de 13.500 personas pertenecientes a pueblos indígenas fueron desplazadas en 2008, es decir, 3.5\% del total de la población desplazada en Colombia; [... mientras que] las mujeres, niñas y adolescentes constituyen la mayoría del

\footnotetext{
${ }^{2}$ Cifras Acción Social: Número de hogares y personas incluidas en el RUPD según periodo, SIPOD (Sistema de Información de Población Desplazada), fechas de corte: 31/07/2010. Acceso en: <www.accionsocial.gov.co/estadísticas>. Cifras CODHES: Víctimas emergentes. Desplazamiento, derechos humanos y conflicto armado en 2008, Boletín informativo del CODHES, № 75, Bogotá, 22 de Abril de 2009, pág. 3.
} 
total de la población desplazada (52\%). Además, la gente sigue huyendo para salvar su vida y preservar su seguridad de los ataques individuales o concurrentes de diversos actores armados como la Fuerza Pública, los nuevos grupos paramilitares o los movimientos guerrilleros [...] En 2008 se reportaron 37 casos de masacres con un total de 119 víctimas. (Codhes, 2009, p. 3 e 4)

El Plan Colombia, creado en 1999, se proponía acabar con el cultivo de la coca, desmantelar los cárteles de la droga y aportar seguridad a la población. Hay resultados colaterales adversos, de acuerdo con Codhes (2009), como la militarización de amplias zonas rurales. Paralelamente, existe la sospecha de que la continuidad de los grupos de paramilitares garantiza seguridad y protección en beneficio de empresas multinacionales y terratenientes.

Todos estos datos confluyen en que, en el ámbito internacional, Colombia ocupe el nivel más bajo de los puestos entre los países en los estudios de monitoreo del desplazamiento interno. Según IDMC ${ }^{3}$, el número de personas desplazadas en América Latina a finales de 2009, ascendía a unos 5 millones de personas, cifra incrementada de manera importante por los desplazados internos que se presentó en Colombia (lo que llevó a ser considerada, junto a Sudán y otros países de África y Oriente Medio, como el país con la mayoría de los desplazados en la actualidad). A partir de un comparativo de las cifras oficiales y de las del CODHES, el documento establece una cifra redondeada entre 3,3 millones y 4,9 millones de personas desplazadas en Colombia. Además, el documento citado menciona las repercusiones del desplazamiento interno en la región, pues muchos de los colombianos que tienen que movilizarse de sus territorios de origen se ven obligados a cruzar las fronteras hacia los países vecinos como Ecuador

\footnotetext{
${ }^{3}$ Internal Displacement Monitoring Center. Relatório Internal Displacement. Global Overview of Trends and Developments in 2009. Disponible en: < http://www.humansecuritygateway. com/documents/IDMC_InternalDisplacement2009.pdf $>$.
} 
El incremento en 2009 es atribuido a las fracasos presentadas en la estrategia del país contra los grupos armados ilegales, lo que condujo a aumentar los desplazamientos internos; además existe una contradicción ante la percepción de la consolidación de los nuevos grupos armados que surgieron tras la desmovilización de las organizaciones paramilitares en 2006, incrementando la violencia en las zonas urbanas (Velásquez; Pinzón, 2008). Todo esto como factores adicionales al enfrentamiento entre las fuerzas militares y los grupos guerrilleros dominantes (FARC, el Ejército de Liberación Nacional ELN y el Ejercito de las Autodefensas Unidas de Colombia - AUC).

En los últimos años, el desplazamiento forzado en Colombia ha estado vinculado a la violencia política y a los enfrentamientos entre grupos armados ilegales, guerrilla, paramilitares; el desplazamiento se ha convertido como una estrategia esencial de control político y militar de los actores sociales en general y en particular a los armados.

Los grupos ilegales buscan ejercer el control sobre territorios estratégicos, por ejemplo, los que cuentan con alta presencia de cultivos ilícitos, o los que constituyen corredores vitales para el contrabando de armas, comercialización de la droga; como también territorios con recursos mineros y energéticos importantes para la economía del país, por ejemplo zonas petroleras, carboníferas, bananeras, entre otros; lo cual realizan ya sea mediante la compra de la tierra en procesos especulativos o mediante la expulsión violenta de sus propietarios (Galindo, 2009). Para ello, emplean amenazas directas hacia la población civil, mediante la ejecución de actos terroristas, la toma de municipios y el reclutamiento forzoso, entre otros, al mismo tiempo el enfrentamiento con otros grupos armados irregulares por el control territorial y con las fuerzas del Estado. Así, la población civil se encuentra en el medio de la confrontación, o es directamente victimizada, careciendo de las garantías mínimas para la protección de su vida e integridad, viéndose forzada a abandonar sus territorios, explicitando los mecanismos de desprotección social (Giraldo, 2007). 
En el modelo capitalista de ocupación del territorio rural, el problema de la tierra representa la variable más importante debido al status y poder que deriva de su posesión. De acuerdo con Galindo (2009), lejos de las estadísticas del desplazamiento forzado por causa del conflicto armado en que la población ha perdido su bien por excelencia, se trata del contexto histórico de la lógica de acumulación capitalista. Así, más que un ejercicio para cuantificar el despojo de tierras y de bienes, el análisis demanda referencias a la violación de los derechos humanos y las incertidumbres que mueven los organismos del Estado Social de Derecho para afrontar el fenómeno y construir una política coherente y efectiva para restituir a las víctimas.

El panorama anterior ha propiciado el surgimiento de varias consecuencias, que impactan de forma negativa a la población en cuestión al generar un detrimento en su bienestar y en sus condiciones de vida. De este modo, se configuran grupos poblacionales hondamente vulnerables y excluidos de los recursos de la democracia y de la ciudadanía (Buendía y Stollbrock, 2007), pues las personas, al ser obligadas a desplazarse de su lugar de origen, se ven inmersas en una situación tal que destruye sus redes sociales y, por veces, sus roles familiares. Eso produce un fuerte desarraigo frente al proyecto original de vida, lo que, a su vez, conlleva a la pérdida de capital humano, condición de inserción social. De este modo, esas personas se insertan en una dinámica en la que las precarias condiciones económicas (tras la pérdida de activos como la vivienda o la tierra) impulsan a contextos de pobreza, desigualdad, exclusión y discriminación (Fleury, 2007). En esta dinámica, envuelven tanto a las comunidades de origen como a las de destino, destruyendo las bases organizacionales que rigen las relaciones sociales cotidianas.

Se señala que durante los últimos años, las principales causas del desplazamiento forzado en Colombia están relacionadas al incremento 
de la actividad armada entre los grupos al margen de la ley para lograr el control de zonas estratégicas para el narcotráfico, tráfico de armas y contrabando; en ello se ven involucrados grupos narcotraficantes, guerrillas y bandas emergentes y criminales tras la desmovilización de las autodefensas; así mismo estos actores vienen incentivando la realización de actos terroristas para contrarrestar las operaciones de erradicación de cultivos ilícitos que viene desarrollando el gobierno nacional. A cada año, al lado de los desplazados, millones de vidas de ciudadanos son perdidas debido a la violencia decurrente del uso de armamentos. Cerca de dos tercios de esa tragedia ocurren en países aparentemente en paz.

\section{El Sistema de Protección Social:} nueva caracterización sobre el gobierno Uribe

La asistencia pública se centra en programas asistenciales ante la persistencia de las desigualdades y de los protestos sociales, que tienen el propósito de suplir las necesidades básicas de aquellos que no cuentan con las capacidades para procurarse los servicios sociales que necesitan a partir de sus propios recursos. Este último componente es normalmente conocido como asistencia social y está centrado en los grupos poblacionales que se encuentran por debajo de la línea de pobreza, que además presentan las más profundas condiciones de vulnerabilidad, bien se trate de menores de edad, adultos mayores, discapacitados, desplazados o población en condición de pobreza en general. La asistencia social puede estar conformada tanto por programas universales como por programas focalizados, pero, en Colombia, este componente del sistema de protección social solamente incluye programas del segundo tipo, los cuales están constituidos por transferencias en efectivo, asistencia en especie y subsidios para servicios. 
Para tal efecto, de acuerdo con lo dispuesto en la Ley 789 de $2002^{4}$, se estructuró el Sistema de Protección Social (SPS), un conjunto de políticas públicas orientadas a disminuir la vulnerabilidad y a mejorar la calidad de vida de los colombianos, especialmente de los más desprotegidos (articulo 1).

El Estado colombiano, durante los ocho años del gobierno de Álvaro Uribe, a partir de los respectivos dos planes de Desarrollo, definió de forma muy retórica las estrategias con el objetivo de lograr que los colombianos tengan igualdad de oportunidades en el acceso y la calidad de un conjunto básico de servicios sociales (Plan Nacional de Desarrollo - PND2006-2010, p. 107); en términos generales, que todos los colombianos tengan acceso a educación de calidad, a una seguridad social equitativa y solidaria, al mercado laboral (promoviendo la formalización o apoyando el emprendimiento) (PND, 2006-2010, p. 107).

Este conjunto de propósitos contempla tres aspectos claves: capacidad de articulación de la oferta de servicios; necesidad de contar con mecanismos de focalización que prioricen las intervenciones a los más necesitados; y posibilidad de integrarse con otros segmentos del mercado (acceso a activos). El SPS se compone de cinco pilares fundamentales en base a los procesos de focalización y priorización de la acciones. En este marco, y teniendo en cuenta que uno de los grandes retos de Colombia es la articulación de las instituciones del SPS y las del mercado laboral (PND, 2006-2010, p. 114), la política de generación de empleo e ingresos se constituye en el principal desafío e instrumento del Estado en la consecución de una equidad social con inclusión social (Fleury, 2007).

Siguiendo esta línea, la Ley 1151 (24/7/2007) por la cual se expide el Plan Nacional de Desarrollo 2006-2010, contiene los compromisos po-

\footnotetext{
${ }^{4}$ Ley 789 de diciembre de 2002, por la cual se dictan normas para apoyar el empleo y ampliar la protección social y se modifican algunos artículos del Código Sustantivo de Trabajo (Artículo $1^{\circ}$ ). Al mismo tiempo la ley expresa una retórica con la cual el nuevo gobierno (Uribe) busca su legitimación social.
} 
líticos referentes a la reducción de la pobreza, atención a los desplazados, promoción del empleo y equidad. El tenor de la legislación comprende que, en las actuales circunstancias históricas, el desempleo, bajo capital social y pobreza extrema están íntimamente asociados. El artículo primero de la ley asegura que, entre los principales objetivos del Plan de Desarrollo, está la apuesta en una política de reducción de la pobreza, promoción del empleo y la equidad, priorizando las poblaciones vulnerables, realizando programas especiales para el empleo en unidades productivas.

En estas circunstancias, uno de los caminos para viabilizar políticas de acción para mitigar mujeres desplazadas es el Convenio de Cooperación, $n^{\circ} 246$, de $2008^{5}$ por las siguientes razones: a) en la medida en que reconoce un amplio conflicto social de alcance nacional con poblaciones en condiciones de vulnerabilidad como son el desplazamiento forzado interno, víctimas y en riesgo de trata de personas, de reclutamiento impuesto, desvinculadas del conflicto y reinsertadas (MPS, 2008, p. 3); b) define obligaciones mutuas para suscitar formas asociativas de generación de ingresos con proyectos o emprendimientos productivos por mujeres cabeza de familia, incluyendo población urbana, rural, afrodescendiente, o población en situación de desplazamiento.

Con el propósito de consolidar el SPS, el Documento CONPES no 3616 de 2009, establece los Lineamientos de la Política de Generación de Ingresos para la población en situación de pobreza extrema y/o desplazamiento, con el intuito de desarrollar sus capacidades y crear opor-

\footnotetext{
${ }^{5}$ Ministerio de la Protección Social, 2008. Convenio de Cooperación N² 246 celebrado entre el Ministerio de la Protección Social y la Organización Internacional para las Migraciones (OIM). Disponible en: <http://www.contratos.gov.co/archivospuc1/2008/C/118001000/0812-80071/C_PROCESO_08-12-80071_118001000_591281.pdf $>$. Esta relevancia es corroborada en la investigación de Esteban Nina. Evaluación ejecutiva al proyecto asistencia técnica a emprendimientos y microunidades productivas de mujeres trabajadoras vulnerables. DPN/ MPS. 2009.Disponible en:< https://sinergia.dnp.gov.co/Sinergia/Archivos/eeaa8e18-3e0a4ed5-ad2c-28947658d75c/MUJERES_VULNERABLES.pdf>.
} 
tunidades para que puedan acceder y acumular activos y, a mediano y largo plazo, alcanzar la estabilización socioeconómica de la población en extrema pobreza y/o desplazada.

\section{Política Social de mitigación para la población en situación de desplazamiento}

El Estado colombiano, dentro del fuero de su actividad, ha venido implementando a partir de 1997 un número creciente de políticas, programas y proyectos enfocados a atender a la población civil víctima del desplazamiento forzado interno. Los programas en cuestión se basan en los lineamientos legislativos y jurisprudenciales que determinan a los mismos, sus objetivos, límites y alcances. El origen de estos se deriva del establecimiento de una Ley 387 (1997) y su legislación complementar y otras leyes gubernamentales como los Planes Nacionales de Desarrollo o las directivas presidenciales - cuando tratan el tema del desplazamiento forzado por la violencia armada.

Pretender esconder lo que ocurre con mujeres y hombres, niñas y niños, que huyen del asesinato, la violación o el reclutamiento forzado, trae como resultados mecanismos de restricción que van en contra de los derechos humanos; de la propia seguridad de los Estados, en la medida que no se incorpora a esta humanidad errante, al Estado mismo, a sus derechos y sus obligaciones (Escobar, 2004, p. 12).

Así pues, la política social de atención a la población desplazada se desarrolla dentro de los lineamientos operativos, procedimentales y jurídicos establecidos por la Agencia Presidencial para la Acción Social y la Cooperación Internacional. El Programa social se había configurado inicialmente con una estructura básica de tres componentes: Prevención, Asistencia Humanitaria y Estabilización Socioeconómica (estos se conser- 
van hasta 2012, pero han sido integrados a un armazón más amplio de Atención Integral de los Derechos de los desplazados).

El primer componente, el de Prevención, representa la respuesta estatal a los hechos generadores de potenciales desplazamientos, con el gran desafío de visualizar y salvaguardar los derechos humanos de la población civil víctima del conflicto armado. Los programas de prevención se expondrán superficialmente debido a que comprenden un componente adicional y diferente a la respuesta estatal a los desplazamientos. La prevención incorpora las dinámicas de seguridad y control territorial del gobierno, a partir de actividades de recuperación de la confianza entre la comunidad y las autoridades, de la gobernabilidad y de la sobrevivencia de las instituciones democráticas (Ibáñez; Velásquez, 2008b; Ibáñez; Vélez, 2005). Medidas adoptadas incluyen el combate a la acción de la guerrilla y a la búsqueda de control de los grupos paramilitares, que pueden ser evaluadas por medio del impacto sobre los desplazamientos.

El segundo componente, Asistencia Humanitaria, se entiende como la atención en el momento inmediatamente posterior al desplazamiento forzado, de acuerdo con el registro en el RUPD, con apoyo alimentario y no alimentario, aseo personal, vestimenta, alojamiento temporal, atención médica y social; información sobre la oferta institucional de servicios y tener acceso a dicha asistencia, tanto en el caso de urgencia como de emergencia; transporte para retornar a su lugar de origen o en busca de un lugar donde residen los parientes. La ayuda recibida bajo este componente se mantendrá solamente hasta un año después de ocurrido el desplazamiento.

Finalmente, el componente de Estabilización Socioeconómica busca contribuir con los procesos de retorno o reubicación en el nuevo espacio y hogares desplazados, brindando posibilidades para que la población satisfaga sus necesidades básicas, materiales y no materiales por medio de su propia gestión o de los programas que para el efecto desarrolle el Estado. 
En suma, se trata de asistir a la población en situación de desplazamiento en los sectores de vivienda, salud, alimentación y educación, en el ámbito de sus propias competencias y de acuerdo con la disponibilidad presupuestal.

El programa pretende ofrecer una respuesta estatal a la situación de la población desplazada, pero son beneficiarias solamente las víctimas que hayan sido inscritas en el RUPD (Registro Único de Población Desplazada), mediante una declaración ante el Ministerio Público, o aquellas que se encuentren en peligro de desplazamiento en el municipio donde residen, además de los colombianos refugiados que se acogen a los planes de repatriación.

En el Plan Nacional de Atención Integral a la Población Desplazada por la violencia (2005) y el documento metas y priorización de recursos presupuestales para atender a la población desplazada por la violencia en Colombia (CONPES, 2005) detallan las intenciones gubernamentales. Desde el año 2006 los desplazados son incluidos como beneficiarios del programa Familias en Acción.

Con el funcionamiento de la asistencia, se constituye paulatinamente la conformación de un Sistema Nacional de Atención Integral a la Población Desplazada (SNAIPD), integrado por 27 entidades. Este tiene como objetivo ofrecer mitigación a los agravantes de la población pobre en situación de desplazamiento, buscando la integración social y económica en los lugares de reubicación (Gutiérrez; Fernandéz, 2009). La transición de la asistencia circunstancial para lo que propone el SNAIPD para el goce de los derechos radica hasta después de años de vigencia de la situación inconstitucional declarada por la sentencia de la Corte Constitucional (T- 025 de 2004), la cual no se ha superado, y hasta que los derechos de la población desplazada hayan sido restablecidos.

Lo que se busca es mitigar a la población asegurando el ejercicio de derechos vitales vulnerados a los hogares y personas afectados, en 
última instancia acogidos por los principios rectores internacionales y las orientaciones de la Corte Constitucional colombiana. Por otra parte, la jurisprudencia expedida sobre el tema está compuesta por un importante número de sentencias de la Corte Constitucional y sus respectivos autos reglamentarios, aclaratorios y de seguimiento; resaltando de manera importante la Sentencia T-025 de 2004 ${ }^{6}$.

En los procesos del sector de la Acción Social y del SNAIPD se establece una ruta de atención según la cual las personas y los hogares desplazados entran inicialmente en una etapa de recepción en las Unidades Territoriales de Acción Social, con un proceso de caracterización por la Ficha Técnica de Evaluación de Capacidades y Necesidades ${ }^{7}$. La información permite identificar los servicios sociales con los cuales las personas se benefician y así la coordinación del SNAIPD puede solicitar los servicios faltantes en el territorio y la remisión a la estrategia Juntos. A través de los programas: Juntos y de Familias en Acción la población desplazada accede inicialmente a la atención de emergencia, siguiendo la gestión para el acceso a los programas regulares de promoción social del Estado (Almanza, 2010).

Las reformulaciones a partir del 2008 tuvieron el propósito de alinear las acciones gubernamentales de Acción Social, las entidades pertenecientes al SNAIPD y el programa de Superación de la Pobreza Extre-

\footnotetext{
${ }^{6}$ Hasta el momento, la Corte ha considerado que el Estado de Cosas Inconstitucional no se ha superado, que los derechos de la población desplazada no han sido restablecidos y que, por lo tanto, mantiene su competencia en el caso, ejerciendo su función jurisdiccional interna y habilitando continuamente escenarios de audiencia pública y debate con la participación de los diferentes actores sociales. (Informe de CODHES, 9 junio 2010). Reforzando los informes de la Comisión de Seguimiento a la Política Pública sobre Desplazamiento Forzado y de sus varios informes a la corte constitucional. Disponible en: <http://www.acnur.org/t3/fileadmin/scripts/ doc.php?file $=$ pais $/$ docs $/ 2815>$.

${ }^{7}$ Fuente: Subdirección de Atención a la Población Desplazada, Agencia de la Presidencia para la Acción Social y la Cooperación Internacional-Acción Social, Disponible en: <http://www. accionsocial.gov.co/contenido/contenido.aspx?catlD =295\&conID=1933> <http:/www. dps.gov.co/contenido/contenido.aspx?catID $=295 \&$ conID $=1933 \&$ pagID $=8778>$.
} 
ma Juntos. El panorama político presionó para que el Estado colombiano reformulase la atención a la población desplazada, soportándolo ahora en busca de indicadores ${ }^{8}$. Hubo cambios en la formalización de las tres líneas de acción conforme el informe del Gobierno Nacional a la Corte Constitucional sobre el avance del Estado de Cosas Inconstitucional (Arcila; Naranjo, 2010): la Prevención y Protección, Atención Integral, y Verdad, Justicia, Reparación y Garantías de no Repetición. Para un proceso continuo de acción y retroalimentación para la reformulación se establecieron cuatro elementos de carácter transversal: enfoque diferencial, capacidad institucional y sistemas de información, participación y articulación territorial.

En el panorama planteado, entre los componentes que caracterizan las políticas para la población desplazada, se establecen como requisito para el acceso al servicio que las carencias de un individuo u hogar sean demostrables, con esto, los desplazados son sometidos a procesos de selección y verificación de requisitos. Además, debido a que los programas asistenciales no han logrado abarcar el total de la población desplazada, cubriendo en parte las demandas de los que presentan las más precarias condiciones de vida, la jurisprudencia expedida por la Corte Constitucional ha recalcado la necesidad de implementar medidas de reconocimiento de la población desplazada y de sus necesidades.

En una investigación en una localidad al sur de Bogotá a respecto de rasgos del problema de desplazamiento, Palacios-Sanabria apunta algunas razones o interrogantes a partir de los derechos sobre la asistencia efectiva:

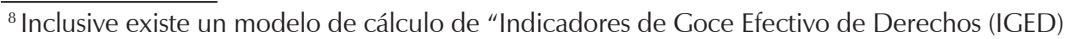
de la población víctima del desplazamiento forzado desde 2008, para verificar el avance o retroceso en la garantía de los derechos. Para conferir parte de estos resultados Caballero y Suarez: disponible en: <http://www.dnp.gov.co/LinkClick.aspx?fileticket $=$ G9Hzz_ olVaA\%3D\&tabid=1296> .
} 
[I]rregularidades y falta de precisión en las bases de datos, la ausencia de un manejo unificado de la información, inexistencia de un lugar de paso para brindar efectiva y oportuna atención a las víctimas del desplazamiento, falta de reconocimiento del fenómeno del desplazamiento urbano y falta de seguimiento a la problemática y a las posibles soluciones (Palacios-Sanabria, 2007, p. 218).

En el tema de los indicadores educativos, es importante resaltar que la situación de los desplazados en este sector es más precaria que la de los hogares e individuos pobres de los municipios receptores. Según Ibáñez y Velásquez (2008a) y también López (2007), los desplazados se encuentran en una condición más precaria que los pobres e indigentes urbanos; la posibilidad de la asistencia educativa de los niños desplazados aumenta en los municipios receptores, debido a la mayor oferta educativa, pero la tasa de asistencia escolar es menor entre los desplazados que para el restante de la población pobre, además de presentar un alto índice de deserción escolar derivada de la discriminación y de la presión para vincularse en actividades generadoras de ingreso. El analfabetismo de los jefes de hogar se presenta igualmente en una proporción más elevada en comparación con los pobres urbanos.

Por otra parte, los desplazados en edad de trabajar nuevamente se encuentran en desventaja debido al reducido valor de su capital social en la ciudad, limitando las posibilidades de inserción en los mercados de trabajo y restringiendo sus opciones de empleo a actividades informales y mal remuneradas. Entonces, uno de los factores determinantes para la superación de la vulnerabilidad es el tema educacional, ya que una optimización en la cobertura y la calidad del mismo hará posible evitar, por un lado, que los individuos caigan en pobreza crónica y, por el otro, que dicha vulnerabilidad tenga un efecto de generaciones. 
Algunos de los indicadores estiman las circunstancias de vulnerabilidad de los desplazados, y toman como ejemplo las cifras referentes al acceso a la educación, básicamente porque este factor: 1) representa la temática con el mayor número de fallos expedidos por la Corte Constitucional, 2) es un componente que concentra la superación de las causas estructurales de la vulnerabilidad, además de romper con el ciclo de pobreza, y 3) porque generalmente en los estudios sobre política social, la educación constituye el sector que presenta mejores resultados en el gasto público social.

Según cifras de Acción Social, con corte a febrero del 2008, la cobertura en educación en 2003 llegaba solamente a 29 mil 707 niños y jóvenes desplazados matriculados en el sistema educativo. Si se supone que, dentro de esa cifra, Acción Social contabiliza los dos rangos de edad propios de la educación preescolar, primaria, secundaria y media - de 6 a 9 y de 10 a 25 - había un desfase entre la oferta institucional y la demanda de 23 mil 863 cupos educativos. Durante los años subsiguientes, la cobertura en educación ha presentado mejoras considerables, que se traducen en un incremento de 93 mil 075 cupos adicionales entre 2004 y 2007. Aun así, este último incremento resulta insuficiente comparado con el del primer lapso, más aún si se tiene en cuenta que el número de cupos alcanzados en cada año hace referencia a un número acumulado de personas insertas en el sistema educativo, del total de desplazados en edad escolar hasta cada uno de esos años y no durante el transcurso de los mismos de manera individual.

Un aspecto importante a evaluar en este punto es que la cobertura en educación alcanzada parece que no resulta idónea en cuanto a la proporción de niños/as atendidos/as que están por fuera del rango de edad para el nivel educativo correspondiente; mientras que las tasas brutas de escolaridad resultan bastante bajas para la educación preescolar, secundaria y 
media. Adicionalmente, el boletín de CODHES Ahora por los Desplazados (2008) establece que las tasas de deserción intra-anual se encuentran en $3.4 \%$ para los niños entre los 5 y 11 años, en 5\% para los que están entre 12 y 15 años, y en $18.5 \%$ para los que están entre 15 y 16 años.

Con miras a examinar los resultados de la Atención Integral para el Goce Efectivo de los Derechos (GED), en el informe de la Comisión de Seguimiento ${ }^{9}$ el componente educativo se encuentra al interior de la etapa denominada de atención básica. Básicamente, es posible evidenciar que la situación no presenta cambios sustantivos durante el periodo 20042010, el cual comprende el periodo sobre el cual el gobierno nacional presenta su informe a la Corte. Así pues, según el documento, entre 2004 y 2010 la población desplazada entre 5 y 17 años de edad atendida por el sistema educativo pasó, según el informe del gobierno, de 111 mil 901 a 557 mil 860 estudiantes. La proporción de atención a la población desplazada sobre población atendida total pasó de 2.1\% en 2006 a 4.6\% en 2009, en los niveles de preescolar, básica y media. No obstante este aumento, dicha proporción todavía resulta inferior a la participación de población desplazada en la población total. Las cifras anteriores son bastante explícitas en torno a tres aspectos primordiales:

1) La política a los desplazados presenta indicadores que claramente demuestran cobertura insuficiente en el sistema educativo. El punto neurálgico es que no solamente se trata de un problema de desfase entre oferta y demanda de la población en edad escolar, sino que además se trata de un descuadre entre rango de edad y nivel educativo correspondiente

\footnotetext{
${ }^{9}$ O Modelo de Atención Integral para el Goce Efectivo de los Derechos (GED) de la Población Desplazada y el componente educativo es examinado en el documento Comentarios al Informe del 1 de julio de 2010 del Gobierno Nacional a la Corte Constitucional Sobre la Superación del Estado de Cosas Inconstitucional Declarado Mediante la Sentencia T-025 de 2004 de la Comisión de Seguimiento a la Política Pública Sobre Desplazamiento Forzado.
} 
2) La política social diseñada e implementada para responder a las necesidades de los desplazados se desarrolla de acuerdo con las limitaciones presupuestales, como establece el Decreto 2569 del año 2000, que dispone que la ejecución de los programas de estabilización socioeconómica depende de la disponibilidad presupuestal.

3) Finalmente, y como conclusión de lo anterior, es posible establecer que el modelo de protección social en Colombia - referente a la población en situación de desplazamiento - está lejos de enfrentar las causas estructurales de la vulnerabilidad, pues las estrategias actúan poco sobre las capacidades humanas y simplemente mitigan las privaciones inmediatas sin que eso implique romper con las incertidumbres de calidad de vida a futuro.

[E]l modelo asistencial no tiene como objetivo final la guarda del bienestar de quienes se ven obligados a desplazarse, sino la contención de su éxodo dentro de las fronteras de los Estados en los que se han originado. Por tanto la suerte de quienes huyen es sacrificable en aras de obtener el fin último que persigue todo el modelo. La política colombiana se ha inspirado en este modelo, lo que permite explicar sus limitaciones para ofrecer una protección integral a los desplazados. (Mojica, 2009, p. 11).

Para entender la política social para la población desplazada en Colombia, hay que tener en cuenta que esta se ha consolidado en torno a su componente de protección social, el cual, debido a la condición de la problemática y al enfoque institucional adoptado para hacerle frente, se ha restringido al principio de asistencia pública, basado en los criterios originados desde los modelos de protección social residual. Dichas políticas son dirigidas a grupos poblacionales que, en su particularidad, se encuentran en situación de vulnerabilidad, lo que los hace diferentes al resto de la población. Esto ha llevado a que las políticas púbicas diseñadas para atender a tales grupos se soporten no en un gasto público social que pre- 
tenda ofertar servicios públicos a la población desde su condición de ciudadano, sino en un gasto en asistencia social, convirtiendo los servicios sociales dirigidos a los más pobres en bienes y servicios degradados o de baja calidad. Los desplazados han sido limitados a un tipo de ayuda basada en programas momentáneos y circunstanciales que se materializan en el enfoque de la asistencia social, los cuales establecen mecanismos de atención especiales para un grupo poblacional, que es considerado como un conglomerado problema. El aspecto a resaltar de esto es que, dentro de dicha lógica,

El acto de recibir un beneficio, aunque tenga carácter permanente, no transforma al individuo en poseedor de un derecho, ya que su concesión dependerá permanentemente del poder discrecional de un funcionario. En ese sentido, la asistencia social tiene más un carácter propiamente preventivo y punitivo que el de una garantía de los derechos de la ciudadanía... (Fleury, 2007, p. 110).

Aunque resulte inapropiado abandonar la importancia que adquiere en muchos escenarios la asistencia social temporal - como es la asistencia y el socorro otorgado en el momento inmediatamente posterior al desplazamiento o frente a la extrema urgencia evidenciada por individuos $\mathrm{u}$ hogar desplazado -, es posible afirmar que dichas políticas no implican soluciones a las causas estructurales de la vulnerabilidad.

Así pues, la protección social basada en la asistencia social residual puede ser concebida como un componente preliminar y una temporalidad previamente limitada, que buscan mitigar el impacto inicial del desplazamiento. El desafío es incorporar en otras políticas que confieran beneficios sostenidos, en aras de mejorar la calidad de vida, con un proceso de la resocialización y como ciudadanos sujetos de derechos. Se trata entonces de construir efectivamente un espiral de atención o atención integral y no una cascada de programas segmentados por tipo de demanda.

El planteamiento de que la política pública para atender a la población desplazada ostenta un problema estructural de diseño y aplicación 
resulta en la implementación de una cascada de programas residuales, que dejan mucho que desear en materia de derechos sociales. Como bien lo establece ACNUR (Alto Comisionado de las Naciones Unidas para los Refugiados):

[...] una de las limitantes que existen para la superación del Estado de Cosas Inconstitucional es la incipiente incorporación del enfoque de derechos en la formulación de la política pública [...] Así, las políticas sobre las cuales se estaría soportando la respuesta estructural del Estado colombiano en materia de desplazamiento no parten estrictamente de un enfoque de derechos, ni están concebidas para atender las necesidades específicas de la población desplazada; excepción hecha de la fase de atención de emergencia (ACNUR, 2007, p. 25).

Resulta preciso clarificar que el enfoque limitado al otorgamiento de bienes y servicios a los vulnerables, de manera de controlar el descontento por la carencia de servicios sociales, es muy distinto del enfoque de derechos en las políticas sociales. El último trata más bien de una cultura y concepción inclusiva con el reconocimiento de los derechos a la ciudadanía, de tal suerte que sea posible materializar los derechos consagrados en la Constitución. El derecho a tener derechos por los individuos desplazados, de acuerdo con Caballero y Suarez (2007) supera así la enorme brecha entre las premisas constitucionales y los déficits en los indicadores de la ejecución de las políticas de combate a las desigualdades.

Resumiendo, la baja eficiencia y eficacia de la política pública para atender a la población desplazada en Colombia gira en torno a cuatro debilidades conceptuales, a saber: 1) la magnificada ponderación otorgada al componente de asistencia social al interior de la política pública para la población desplazada; 2) la diferencia existente entre el gasto público enfocado a lo social y el gasto público enfocado a la asistencia social; 3) la inconsistencia entre la adopción de una lógica asistencialista temporal 
y sectorizada, y la necesidad de adoptar medidas que hagan frente a las problemáticas estructurales que determinan la condición de vulnerabilidad de la población, a partir de programas secuenciales con enfoque de derechos, que validen la ciudadanía y procuren mejores niveles de calidad de vida; finalmente, y en relación con el punto anterior, 4) el problema de diseño y aplicación de la política de atención a la población desplazada, en la medida en que no parte de las necesidades reales de los ciudadanos, al considerarlos objetos de política, y no sujetos de las mismas.

\section{Consideraciones finales}

En su desarrollo, el artículo examina la consolidación de una infraestructura institucional y sus limitaciones presupuestales, con un gasto público social que se ha enfocado y ha sido limitado a la asistencia social. También, se está consolidando una institucionalidad informal permisiva, que además de no tener espacios consolidados para evaluar los resultados de las políticas y exigir rendición de cuentas de sus gobernantes, se encuentra poco informada no sólo en materia de derechos, sino también en cuanto a la oferta institucional y en lo que a la gestión gubernamental se refiere; lo que implica per se el debilitamiento de la democracia, de su sistema político y del Estado Social de Derecho.

Las circunstancias de la población desplazada significan una ruptura del contrato social, fundamento de la sociedad moderna y de los lazos sociales en que los individuos se insieren (Escobar, 2004); de esta forma provocando fenómenos de desafiliación, desvinculación y de ruptura en las relaciones de alteridad o desarraigo social. .

Lo interesante del enfoque de derechos es la validación de un derecho, al que todos los individuos tienen acceso en su condición de ciudadanos y como ratificación de la ciudadanía social. En las políticas con 
enfoque social, según plantea Palacios-Sanabria (2007, p. 197) [...] se da gran despliegue a los derechos económicos, sociales y culturales y a su materialización, toda vez que las finalidades del Estado se resumen en alcanzar el máximo de satisfacción de los derechos de aquellos que hacen parte del conglomerado social.

La implantación de una política social desde el enfoque de derechos implica mucho más que el uso del discurso político - implica la transición de los derechos humanos a los derechos sociales efectivos, y su internalización. Así, los servicios de asistencia pública per se son derechos sociales que hacen parte de la ciudadanía, dejando de lado ese discurso de beneficiarios, individuos ajenos al proceso de construcción del bienestar.

A lo largo de la exposición ha sido posible corroborar que existe un problema estructural en las políticas sobre las cuales se soporta la respuesta estatal, y una de las razones es la manera en que los programas de atención han sido pensados, diseñados e implementados. Al soportarse en un ordenamiento irregular - que además de generar una resistencia desarticulada sobre las causas estructurales de la vulnerabilidad de las personas, impidiendo la existencia de un proceso evolutivo en la atención-, hace que cada una de las acciones encaminadas a atender la condición de vulnerabilidad se encuentren orientadas hacia un norte homogéneo que no tiene en cuenta las particularidades de cada caso. También, dichos programas son establecidos de manera precipitada, sin contar con lineamientos horizontales ni transversales, al estar determinados únicamente por la disponibilidad presupuestal en un momento determinado. Entonces, y debido a lo anterior, se privilegian los programas sectoriales no como complemento de los esquemas y planes de acción establecidos desde el gobierno nacional y prolongados a los gobiernos departamentales y municipales de manera independiente, sino como los programas centrales, que al solucionar solamente una parte de determinada problemática, desconocen la integralidad de los individuos como sujetos de política. 
En el problema de burocratización de lo social se dejó de lado la visión del sujeto social - entendido como colectividades específicas que tienen una serie de elementos comunes los cuales permiten construir una identidad - para la identificación de las demandas sociales, desde un conjunto de instituciones que terminaron por convertir el reconocimiento y cumplimiento de los derechos en una concepción desprovista de la visión integral de los sujetos sociales que constituyen tal demanda. Entonces, implementar el enfoque de derechos en la reforma de la política social es erradicar la cascada de programas focalizados y permitir entrar en la lógica de una espiral de atención con programas secuenciales, modificando el papel del Estado en la protección social. Una estructura de tal índole implica etapas que se deben superar para pasar al siguiente eslabón de atención, de tal suerte que los beneficiarios se esfuercen por alcanzar los eslabones subsiguientes.

Aloisio Ruscheinsky. Doutor en sociología, profesor de posgrado en la Universidad Unisinos, São Leopoldo, Brasil, tiene interese en investigaciones en desigualdades, actores sociales y medio ambiente.

$\measuredangle$ aloisior@unisinos.br

Esteban Arnoldo Nina Baltazar. Filósofo boliviano; magister en Economía, Pontificia Universidad Javeriana, magister en Planificación en Desarrollo Regional, Universidad de los Andes. Actualmente es profesor investigador y director de la Maestría en Política Social de la Facultad de Ciencias Políticas y Relaciones Internacionales de la Pontificia Universidad Javeriana de Bogotá.

$\triangle$ enina@javeriana.edu.co 


\section{Referencias}

ACCIÓN SOCIAL, Guía de Atención Integral a la población desplazada por la violencia en Colombia. Convenio Acnur-Acción Social. Bogotá, marzo de 2008. Disponible en: <www.accionsocial.gov.co $>$.

ACNUR. Balance de la Política Pública de Prevención, Protección y Atención al Desplazamiento Interno Forzado en Colombia, 2002-2004. Bogotá, 2004. Disponible en: <http://www.acnur.org/paginas/index.php?id_pag=3109>.

ACNUR. Introducción, Conclusiones y Recomendaciones del Balance de la Política Pública de atención integral a la población desplazada por la violencia 2004-2006. Bogotá, 2007. Disponible en: <http://www.acnur.es/ PDF/4901_20120402135811.pdf >.

ACNUR. La protección internacional de refugiados en las Américas. Quito, Ecuador 2011. Disponible en: <http://www.acnur.org/t3/fileadmin/Documentos/ Publicaciones/2012/8340.pdf?view $=1>$.

ALMANZA, J. M. O. Mujer no, madre: Análisis crítico del impacto generado por el Programa Familias en Acción en madres beneficiarias del Barrio Jerusalén de Bogotá. 2010. Maestria En Política Social, Pontificia Universidad Javeriana, Bogotá, 2010.

ARCILA, A. M. G.; NARANJO, D. M. Q. Política pública de atención a población desplazada: perspectiva de la atención humanitaria. Diálogos de Derecho y Política. Universidad de Antioquia, oㅡ 5, 2010.

BARRIOS, E. V. Dimensiones actuales de lo público. A propósito de las interrelaciones entre Estado, Management y Sociedad. Pensamiento y gestión, n. 18, p. 37-68, 2005.

BUENDÍA H. G.; STOLLBROCK, G. La democracia y el conflicto armado en Colombia. In: CALDERÓN, F. (Coord). Ciudadanía y desarrollo humano. Cuaderno de Gobernabilidad Democrática I. Buenos Ayres: PNUD y Siglo Vientiuno, 2007, p. 297-316.

CABALLERO, C.; SUAREZ, A. Indicadores sobre Desplazamiento Forzado en Colombia desde la mirada del Estado. In: LÓPEZ, M. S. (ed). Evaluación del Impacto de Intervenciones sobre el Desplazamiento Forzado Interno. Hacia la Construcción de un Î́ndice de Realización de Derechos. Bogotá: Consejería en Proyecto (PCS), 2007, p. 141-163.

CODHES INFORMA. Ahora por los Desplazados. Boletín de la Consultoría para los Derechos Humanos y el Desplazamiento. Bogotá, 05 feb. 2008. Disponible en: < www.codhes.org $>$. 
CODHES INFORMA. Victimas emergentes. Desplazamiento, derechos humanos y conflicto armado en 2008. Boletín no 75, Bogotá, 22 abr. 2009. Disponible en: $<$ www.codhes.org $>$.

CONPES (Consejo Nacional de Política Económica y Social). Metas y priorización de recursos presupuestales para atender a la población desplazada por la violencia en Colombia, Bogotá, no 3400, 2005.

CONPES (Consejo Nacional de Política Económica y Social) . Lineamientos de la política de generación de ingresos para la población en situación de pobreza extrema y/o desplazamiento. Bogotá, no 3616, 2009. Disponible en: < http://www. dnp.gov.co/LinkClick.aspx?fileticket $=$ Ullc8o2yuyo\%3D\&tabid=907> .

ESCOBAR, A. Desplazamientos, desarrollo y modernidad en el pacifico colombiano. In: RESTREPO, E.; ROJAS, A. Conflicto e (in)visibilidad: Retos en los estúdios de la gente negra en Colombia. Popayán: Universidade del Cauca, 2004, págs. 53-72.

FERNANDEZ, A. N. ¿̇Independencia o control? Los derechos sociales y los esfuerzos del ejecutivo por el control de la corte constitucional en Colombia. Revista de Estudios Políticos, no 143, p. 129-161, 2009.

FLEURY, S. Los patrones de exclusión e inclusión social, in CALDERÓN, Fernando (coord). Ciudadanía y desarrollo humano: cuaderno de gobernabilidad democrática. Buenos Aires: Siglo XXI, 2007, p. 97-122.

GALINDO, W. G. El desplazamiento forzado y el despojo de la tierra efectos de un modelo capitalista de producción en Boyacá. Periodo 1997-2007. Apuntes del CENES. Bogotá, v. 28, n. 47, p. 133-154, 2009.

GIRALDO, C. ¿Protección o Desprotección Social?, Bogotá: Ediciones desde abajo, 2007.

GUTIÉRREZ, D. F.; FERNANDÉZ, A. Proyecto de Vidas Móviles: caracterización de las poblaciones en situación de desplazamiento y vulnerable receptora en la localidad de Ciudad Bolívar. Bogotá: Universitas Medicas, v. 50, p. 41-57, 2009. IBÁÑEZ, A. M.; MOYA, A. La Población Desplazada en Colombia: Examen de sus condiciones socioeconómicas y análisis de las políticas actuales. Bogotá. Departamento Nacional de Planeación, 2007, p. 1-141.

IBÁÑEZ, A. M.; QUERUBÍN, P. Acceso a Tierras y Desplazamiento Forzado en Colombia. Bogotá: Documento CEDE, Universidad de los Andes, n. 23, 2004, $114 \mathrm{p}$.

IBÁÑ̃Z, A. M.; VELÁSQUEZ, A. El proceso de identificación de víctimas de los conflictos civiles: una evaluación para la población desplazada en Colombia. Bogotá, Documento CEDE, n. 36, 2006. 
IBÁÑEZ, A. M.; VELÁSQUEZ, A. El impacto del desplazamiento forzoso en Colombia: condiciones socioeconómicas de la población desplazada, vinculación a los mercados laborales y políticas públicas. CEPAL, Santiago, n. 145, 2008a.

IBÁÑEZ, A. M.; VELÁSQUEZ, A. La política pública para atender a la población desplazada: ¿Cuáles deben ser las funciones de las autoridades locales? Universidad De Berna, Washington, 2008b.

IBÁÑEZ, A. M.; VÉLEZ, C. E. Civil Conflict and Forced Migration: The micro determinants and the welfare losses of displacement in Colombia. Documento CEDE, n. 35, Universidad de los Andes, 2005.

LÓPEZ, M. S. (ed). Evaluación del Impacto de Intervenciones sobre el Desplazamiento Forzado Interno. Hacia la Construcción de un Índice de Realización de Derechos. Bogotá: Consejería en Proyecto (PCS), 2007.

MOJICA, B. Cuando los derechos son la jaula. Trasplante rígido del soft law para la gestión del desplazamiento forzado. Estudios Políticos, 35, Instituto de Estudios Políticos, Universidad de Antioquia, Medellín, 2009, p. 11-32.

PALACIOS SANABRIA, María Teresa. Política Pública con Enfoque en Derechos Sociales: realidad o ficción para la población desplazada en la localidad de Usme. Estudio Socio-jurídico, Bogotá, v 9, p. 190-232, 2007.

PLAN NACIONAL DE DESARROLLO (PND). 2006-2010. Estado Comunitario: Desarrollo para todos. Disponible en: <http://www.dnp.gov.co/PND/ PND20062010.aspx>.

VELÁSQUEZ, A. V.; PINZÓN, V. G. Violencia urbana, seguridad ciudadana y políticas públicas, la reducción de la violencia en las ciudades de Bogotá y Medellín.

Pensamento Iberoamericano, n. 2, p. 249-270, 2008.

VELÁSQUEZ, F. E. C. (coord.). Política social en Colombia: equidad territorial, relaciones intergubernamentales y participación ciudadana. Un análisis del programa familias en acción. Bogotá: Fundación Foro Nacional por Colombia/ Ford F., Informe de avance № 1, 2011. 